\title{
Administrative Workload of GP's in the Republic of Macedonia: an Urgent Call for Change
}

\author{
Vladimir Lazarevik, MD, MPH \\ Institute of Social Medicine, Medical Faculty, Ss. Cyril and Methodius University in Skopje, Skopje, Republic of Macedonia
}

\footnotetext{
Citation: Lazarevik V. Administrative Workload of GP's in the Republic of Macedonia: an Urgent Call for Change. Maced J Med Sci. 2012 Jul 31; 5(2):194-196. http://dx doi.org/10.3889/ MJMS.1957-5773.2012.0227.

Key words: administrative workload; general practitioners; continuity of care; referals; Macedonia.

Correspondence: Dr. Vladimir Lazarevik. Institute of Social Medicine, Medical Faculty, Vodnjanska 17. Skopje 1109 Macedonia. Phone: +389 3125 310. E-Mail: vlazarevik@healthgrouper.com

Received: 30-Jan-2012; Revised: 12-Feb-2012; Accepted: 29-Feb-2012; Online first: 12-Jun-2012

Copyright: @ 2012 Lazarevik V. This is an open access article distributed under the terms of the Creative Commons Attribution License, which permits unrestricted use, distribution, and reproduction in any medium, provided the original author and source are credited.

Competing Interests: The authors have declared that no competing interests exist.
}

\section{Abstract}

An email survey was conducted among general practitioners in the Republic of Macedonia to assess their self reported administrative workload and communication with specialists. More than $56 \%(55.8 \%)$ of the surveyed physicians reported that they spent over $40 \%$ of the time with patients to administrative procedures, follow by poor communication with specialists after the referral. Urgent reforms are needed to advance the work of GP's and improve referral communication with specialists in order to upgrade the quality of the services they provide to their patients.
Many countries are faced with rapid changes and reforms in their health care systems. These changes are directly reflected over the workload of physicians, and quality of care they provide to the patients [1]. The cost containment of scarce health care resources and further administrative measures imposed over the work of physicians are among the key contributors to increase their administrative workload. The aspect of administrative workload of general practitioners has been strongly associated with decreased job satisfaction [2]. Furthermore, physicians' satisfaction has been associated with prescribing behavior, patient satisfaction and quality of care in general [3-5].

A rapid email survey was conducted among general practitioners working in the Republic of Macedonia. Email addresses of physicians were made available from www.healthgrouper.com, an online provider of comprehensive information for physicians and health care providers. The study population included physicians from all parts of the country. In total 135 responded to the survey. Of the 119 physicians who 
started the survey, 96 physicians fully completed the questionnaire and submit their responses. The questionnaire covered four main dimensions of selfreported physicians work: workload, administration, referral practices and communication with specialists.

In total 54 doctors or $56 \%$ reported that on average it takes them more than $40 \%$ of their time with patients to complete the administrative procedures. Moreover, 28 or $29.5 \%$ of the surveyed physicians reported that the administrative workload takes more than $30 \%$ of their time with the patients (Figure 1).

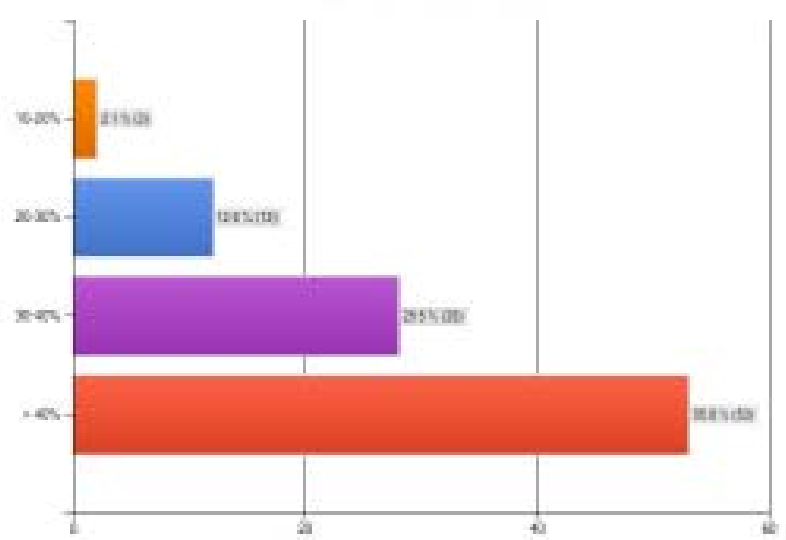

Figure 1: What percentage of the time spent with the patient you devote to administrative procedures (prescriptions, referrals, etc)?

The majority of the physicians reported that it takes 15 minutes to complete one regular examination with the patient. Thus, it is estimated that on average administrative procedures take over 6 minutes of the time a physician is spending with the patient. After the completion of the medical examination, some of the patients were referred to specialists for further assessment and care. On average, 46 or $48.4 \%$ of the primary health care physicians stated that they refer 1 to 5 patients to specialists per day; an additional 34 or $36 \%$ stated that they refer 6-10 patients. The remaining 15 physicians or $16 \%$ of those responding reported that they refer more than 11 patients to specialists examination. This question was supplemented with possibility for the physicians to add comments. Many doctors reported that the increased number of referrals to specialists was due to the administrative requirements of the Health Insurance Fund to request specialist opinion when prescribing certain medications.

Several questions of the survey addressed the process of how patients schedule specialist appointments, and communication between the general physicians and the specialist to follow patient's health condition. In scheduling a specialist appointment, 58 or $61 \%$ of the physicians stated that there is no system how to schedule an appointment. An additional 35\% stated that the patients themselves scheduled the appointments.

The most worrying finding was that even $82 \%$ of the surveyed physicians reported that there is no additional communication with the specialist after the referral is issued; $85 \%$ of the doctors stated that the only way to follow patient's condition is if the patient returns to them (Figure 2). Then the GP can follow up the patient's condition through the administrative documents. A few doctors reported that there is informal communication between doctors by phone or some other way.

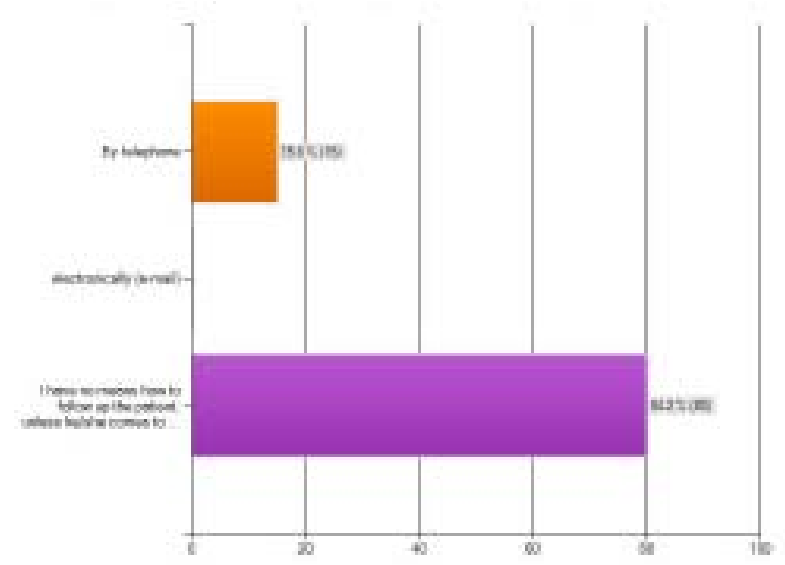

Figure 2: How do you follow up the patient after is referred to specialists?

The results of our survey raise concerns and open questions that require serious debate in the health policy and academic circles. Firstly, there is an obvious need for the health authorities to find ways how to decrease the administrative workload of general practitioners if they wish to improve their efficiency and quality of care GP's provide to their patients. Secondly, there is urgent need to address the issue of poor communication between the primary health care physicians and specialists. In order to provide integrated care to the patients there should be continuity in the process of whereby the primary health care physician can follow up the health condition of the patient throughout the whole process of medical care. The clear gap in communication can be easily solved using the modern communication means such as Internet. The ministry of 
health and health insurance fund should address these issues in more depth in order to improve the quality and efficiency of care.

This survey has certain limitations since it was conducted among primary health care physicians for which Healthgrouper Research Unit had available and valid email addresses. Findings of this survey should be confirmed and supplemented by additional aspects or refuted by further studies among primary health care physicians in the Republic of Macedonia.

\section{References}

1. Bovier PA, Perneger TV. Predictors of work satisfaction among physicians. European Journal of Public Health.
2003;13:299-305.

2. Van Ham I, Verhoeven AA, Groenier KH, Groothoff JW, De Haan J. Job satisfaction among general physicians: A systematic literature review. European Journal of general practice. 2006;12:174-180.

3. DiMattero MR, Sherbourne CD, Hays RD, et al. Physicians' characteristics influence patients' adherence to medical treatment: results from the Medical Outcomes Study. Health Psychology. 1993;12(2)93-102.

4. Haas JS, Cook EF, Puopolo AL, Burstin HR, Cleary PD, Brennan TA. Is the professional satisfaction of general internists associated with patient satisfaction? J General Internal Medicine. 2000;15(2):122-8.

5. Kassirer JP. Doctor discontent. N End J Med. 1998;339(21)1543-5. 